\title{
Torsion of the Fallopian tube in a premenarchal girl
}

\author{
Terence J. Duffy \\ F.R.C.S.
}

University Department of Surgery, Addenbrooke's Hospital, Hills Road, Cambridge CB2 2QQ

\begin{abstract}
Summary
A case of isolated torsion of the normal Fallopian tube, masquerading as acute appendicitis, in a premenarchal girl is presented. The aetiology is considered and the literature reviewed.
\end{abstract}

\section{Introduction}

Torsion of the Fallopian tube is an uncommon condition which presents to surgeons and gynaecologists as an abdominal emergency; it is most commonly mis-diagnosed as acute appendicitis, the correct diagnosis being reached at laparotomy. Torsion of the Fallopian tube alone in a premenarchal patient is much rarer, with only 6 cases previously reported.

\section{Case report}

A 13-year-old schoolgirl was admitted with a 2-day history of abdominal pain and vomiting. The pain was sudden in onset and initially felt in the right iliac fossa, radiating through to the back on the right side and down into the right groin; it was sharp, severe and continuous in nature, and was aggravated by lying flat, or any movement. The pain had gradually become worse during the 2 days before admission and had spread to the whole of the lower abdomen during the preceding $24 \mathrm{hr}$. She was anorexic, had vomited twice and had noted frequency of micturition accompanied by exacerbation of the pain on each occasion. Similar episodes of pain, not so severe as this episode, had occurred intermittently for 2 months. The patient was premenarchal and a virgin.

On examination she looked unwell, was pyrexial at $37 \cdot 3^{\circ} \mathrm{C}$ and had marked oral foetor. The pulse rate was 84 per min. She was grossly obese at $86 \mathrm{~kg}$. Abdominal examination revealed marked tenderness with guarding in the right iliac fossa. Rectal examination revealed marked tenderness on the right side where a bulky swelling was palpable. A provisional diagnosis of acute appendicitis was made and she was prepared for laparotomy. At operation $300 \mathrm{ml}$ of straw-coloured fluid was found lying free in the peritoneal cavity. There was a gangrenous, cystic swelling of the distal half of the right Fallopian tube with a clockwise torsion of 2 complete turns $\left(720^{\circ}\right)$ of the mesosalpinx. The fimbrial end of the cystic and twisted Fallopian tube was closed and would not admit a probe. The proximal half of the right Fallopian tube, the right ovary and left Fallopian tube and the uterus were normal, as was the appendix. The infarcted cystic torsion of the Fallopian tube was excised, appendicectomy was carried out and the abdomen closed. Her postoperative course was uneventful and she was discharged on the fifth postoperative day.

The excised specimen was a grossly distended Fallopian tube measuring $8 \times 6 \times 3 \mathrm{~cm}$ with intense hyperaemia of the serosal surface. Histological examination revealed vaso-dilatation, haemorrhage and inflammatory infiltration of the Fallopian tube.

\section{Discussion}

Torsion of the Fallopian tube was first described by Bland-Sutton (1890) in a patient with hydrosalpinx. Torsion of the Fallopian tube in a premenarchal virgin is a rarer occurrence. Blum and Sayre (1937) in a review of previous publications found only 3 other cases while reporting one of their own and Barnes, Schantz and Shochat (1977) and Evans (1978) have each reported one case.

The mechanism of torsion of a previously normal Fallopian tube is not understood. Numerous mechanisms which may predispose to torsion were considered by Blum and Sayre, including malformations of the tube or mesosalpinx, excessive length, tortuosity, the presence of hydatids of Morgagni, and long veins in the mesosalpinx. The Selheim theory (Blair, 1962) proposes that acceleration/deceleration of the body produces torsion.

When torsion of the Fallopian tube is discovered a predisposing cause, such as underlying pathology or congenital abnormality, should be sought. The contralateral tube and ovary should always be inspected in case a bilateral abnormality is present.

Conservation of the affected tube should be practised where this is possible: Blair (1962) reports 
bilateral patent tubes on salpingography and subsequent normal pregnancy in a woman in whom the twisted tube was saved.

\section{Acknowledgment}

I wish to thank Mr W. A. B. Smellie for allowing me to report this case under his care.

\section{References}

Barnes, W.S., Schantz, J.C. \& Shochat, S.J. (1977)
Torsion of the Fallopian tube in a premenarchal female patient. American Journal of Diseases of Children, $131 \bar{Z}$ 1297.

BlaIR, C.R. (1962) Torsion of the Fallopian tube. Surgeryc Gynecology and Obstetrics, 114, 727.

BLAND-Sutron, J. (1980) Salpingitis and some of its effects Lancet, ii, 1146, 1206.

Blum, L.L. \& SAYRe, B.E. (1937) Torsion of the Fallopiar tube in a virgin. Archives of Surgery, 34, 1032.

Evans, J.P. (1978) Torsion of the normal uterine adnexa in premenarchal girls. Journal of Pediatric Surgery, 13, 195. ¿ 